\title{
Repeated Migration of a Fusion Cage after Posterior Lumbar Interbody Fusion
}

\author{
Jun Gue Lee, Sung Myung Lee, Seok Won Kim, Ho Shin \\ Department of Neurosurgery, College of Medicine, Chosun University, Gwangju, Korea
}

Although posterior lumbar interbody fusion (PLIF) is a widely accepted procedure, perioperative and postoperative complications are still encountered. In particular, cage migration can result in severe sequelae, and revision surgery is technically demanded. Here, we report a rare case of repeated migration of a fusion cage after PLIF. To the best of our knowledge, no report has been previously issued on repeated migration of a fusion cage after PLIF. The authors discuss the radiological and clinical findings of this unusual complication with a review of the literature.

Key Words: Cage $\cdot$ Migration $\cdot$ Complication

\section{INTRODUCTION}

Cage migration interfere with successful fusion in patients undergoing posterior lumbar interbody fusion (PLIF) and causes low back pain or neurologic deficits because the migrated cage compresses neural elements in the lumbar spinal canal. Removal of the migrated cage is necessary for the relief of these symptoms. However, revision surgery for cage migration is technically demanding ${ }^{4,8)}$. Posterior migration of a cage after PLIF procedures has been reported by several authors ${ }^{2,7)}$. In their reports, the risk factors for cage migration mainly include lack of posterior instrumentation and total facetectomy. Both studies supported the role of additional posterior instrumentation in preventing cage retropulsion. Although several reports of posterior cage migration after PLIF or transforaminal lumbar interbody fusion (TLIF) have been published, no report has been reported on the repeated migration of a fusion cage. Here, we report a unique case of repeated posterior migration of a fusion cage after PLIF in addition to posterior instrumentation.

\section{CASE REPORT}

A 54-year man had experienced disabling right leg pain

- Received: March 15, 2013 - Revised: March 17, 2013

- Accepted: March 23, 2013

Corresponding Author: Seok Won Kim, MD

Department of Neurosurgery, School of Medicine, Chosun University,

588, Seosuk-dong, Dong-gu, Gwangju-city 501-717, Korea

Tel: +82-62-220-3126, Fax: +82-62-227-4575

E-mail: ns64902@hanmail.net/chosunns@chosun.ac.kr

*This study was supported by research funds from Chosun University Hospital 2010 with claudication for 12 months before the surgery. Comprehensive conservative treatment failed to improve his symptoms. Radiological studies, including simple radiographs and magnetic resonance imaging (MRI) revealed right foraminal stenosis at the L5-S1 level. Preoperative laboratory tests and bone marrow densitometry (BMD) results were all within normal ranges. He underwent L5-S1 PLIF using Stryker titaniumthreaded cages (length $25 \mathrm{~mm}$, height $7 \mathrm{~mm}$ ) with autogenous local bone chips following posterior instrumentation. Radiographs obtained immediately after surgery showed appropriate positioning of the fusion cages (Fig. 1), and presenting symptoms were much improved. From second postoperative day, the patient started to walk without the leg pain. However, at 10 days after the surgery, he complained of the sudden onset of pain radiating to right thighs and numbness in right leg. Neurologic examination revealed no neurologic deficits, and bowel and bladder function were normal. However, radiographs of the lumbar spine showed posterior migration of the right cage causing significant neural root compression (Fig. 2). Revision surgery was performed to advance the migrated right cage anteriorly, using the same approach as that used during
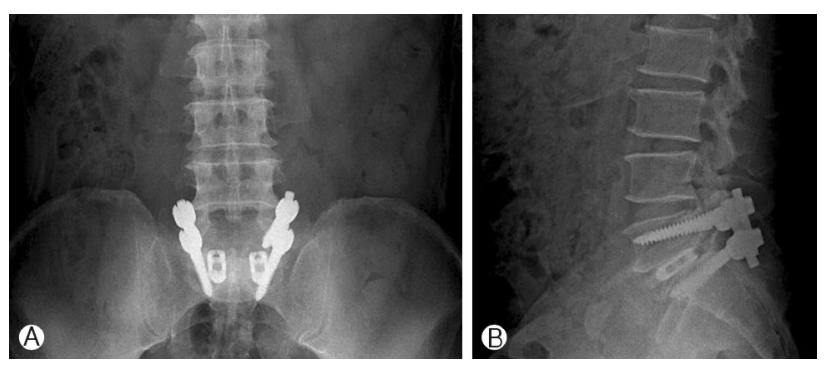

Fig. 1. Postoperative simple radiographs obtained immediately after surgery show appropriate positioning of fusion cages. 


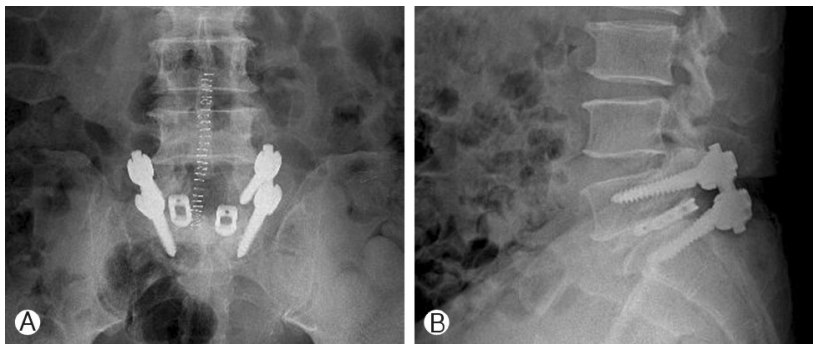

Fig. 2. Simple radiographs taken 10 days after initial surgery reveal posterior migration of the right cage.
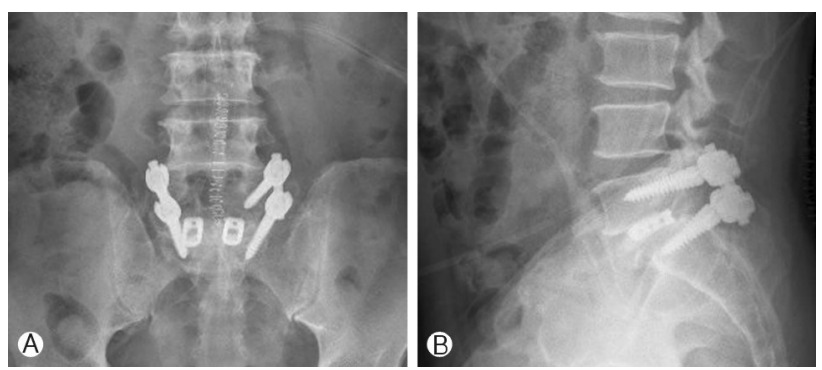

Fig. 3. Simple radiographs after revision surgery show adequate repositioning of the right fusion cage.

the initial surgery (Fig. 3). The right leg pain was relieved immediately after the revision surgery, and plain radiographs showed adequate re-positioning of the cage. After removal of a suction drain at third day, he was allowed to ambulate in a brace. However, two weeks after the revision surgery, the right leg pain gradually worsened. Plain radiographs showed repeated posterior migration of the right cage (Fig. 4). During second revision surgery, after removing both cages, larger cages (length $25 \mathrm{~mm}$, height $9 \mathrm{~mm}$ ) were inserted into the disc space, the screws were replaced with larger diameter screws, and compression maneuver of screw system was applied to prevent further cage migration. The remained graft bone and allograft bone chips were packed into the disc space before inserting the fusion cages. A dramatic improvement in the right leg pain was achieved immediately after the secondary revision surgery. At six month follow-up, he was symptom free and plain radiographs showed inevitable positioning of the cage (Fig. 5).

\section{DISCUSSION}

PLIF is a reliable treatment option in patients with degenerative lumbar spinal diseases, and provides spinal stabilization in balanced alignment, disc height restoration, and mechanical decompression of neural elements ${ }^{6}$. The adoption of titanium interbody cages resolved the problems arising from autograft harvesting. Furthermore, these cages provide immediate stabi-

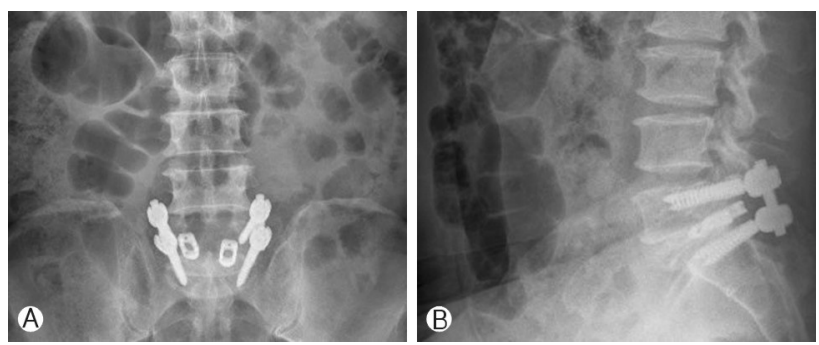

Fig. 4. Simple radiographs taken 2 weeks after revision surgery reveal repeated posterior migration of the right cage.
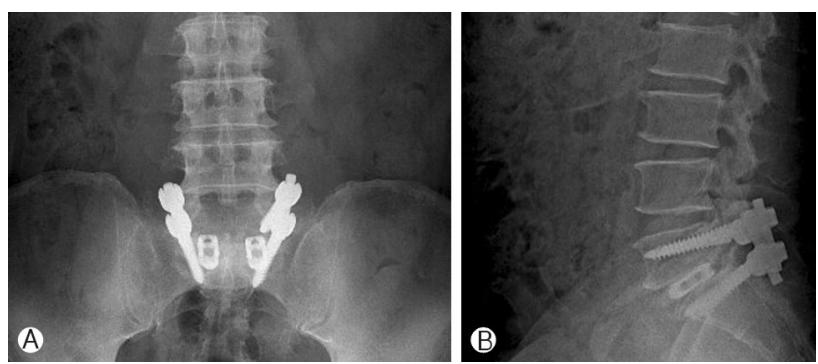

Fig. 5. Simple radiographs taken 6 months after second revision surgery show that appropriate re-positioning was maintained.

lity to spinal levels, restoration of the disc space and neuroforaminal, and a larger surface area, and thus, better fusion rate ${ }^{3)}$.

Although current PLIF procedures are the result of considerable development, some cage-related complications, such as, cage migration or subsidence, may occur. However, few cagerelated complications have been reported. Brodke et al. ${ }^{1)}$ and Lund et al..$^{5}$ in two separate studies, biomechanically tested the initial stability of PLIF alone and with additional posterior instrumentation. Both studies supported the role of additional posterior instrumentation in terms of preventing cage retropulsion, markedly increasing stiffness in axial compression, and more importantly, in flexion-extension torque ${ }^{1,5)}$. Uzi et al. $^{7}$ reported 3 cases of cage migration after PLIF in the absence of posterior instrumentation ${ }^{7}$. They reported retropulsion occurs during spinal flexion, and suggested that this can be prevented by supplemental posterior instrumentation. However, in our patient, the inserted titanium cage was not a standalone device. Kimura et al. ${ }^{4)}$ reported that the risk factors of cage retropulsion after PLIF are; (1) a multilevel fusion procedures, (2) the involvement of L5/S1, (3) greater range of motion (ROM) of the disc space $\left(>10^{\circ}\right.$ on lateral radiographs), and (4) taller discs (>10 $\mathrm{mm}$ at the midpoint of end plates on computed tomographic scans). In this previous study, no case of cage retropulsion after single-level PLIF was observed. However, despite single level PLIF, our patient experienced repeated cage migration at the L5-S1 level, suggesting that the inserted cages did not fit the shape of L5/S1, which 
has a greater disc angle than other disc levels. Furthermore, pedicle screw fixation at $\mathrm{S} 1$ is less rigid than at other spinal locations because of the anatomical nature of the sacrum. Another feasible mechanism of cage migration in our patient concerned a pear-shaped disc space, as a wide anterior space makes it difficult to contact the two anterior points of the cage. In our opinion, as much graft bone as possible, in addition to the cages, should be packed into the disc space at initial surgery to reduce cage movement and facilitate bone fusion. In our patient, we unexpectedly experienced posterior migration of the cage at 10 days after PLIF. This case cautions care must be taken during cage insertion to avoid the possibility of cage migration, and that when unexpectedly encountered, larger cages should be used and more compressive force be applied by pedicle screws to prevent repeated cage migration. Furthermore, it should be noted that repositioning by simply punching cages did not prevent repeated cage migration in our patient. However, more studies about the angle of cages and intraoperative position of the patients are necessary.

\section{CONCLUSION}

We report a case of unexpected repeated cage migration after PLIF. Larger cages and more compressive force should be applied to avoid this unexpected complication.

\section{REFERENCES}

1. Brodke DS, Dick JC, Kunz DN, McCave R, Zdeblick TA: Posterior lumbar interbody fusion: a biomechanical comparison, including a new threaded cage. Spine 22:26-31, 1997

2. Chen L, Yang H, Tang T: Cage migration in spondylolisthesis treated with posterior lumbar interbody fusion BAK cages. Spine 30:2171-2175, 2005

3. Kim HJ, Cho KH, Shin YS, Yoon SH, Cho KG: Minimally invasive posterior lumbar interbody fusion with threaded fusion cage (TFC). J Korean Neurosurg Soc 30:247-253, 2001

4. Kimura H, Shikata J, Odate S, Soeda T, Yamamura S: Risk factors for cage retropulsion after posterior lumbar interbody fusion: analysis of 1,070 cases. Spine 37:1164-1169, 2012

5. Lund T, Oxland TR, Jost B, Cripton P, Grassmann S, Etter C, et al: Interbody cage stabilization in the lumbar spine: biomechanical evaluation of cage design, posterior instrumentation, and bone density. J Bone Joint Surg Br 80:351-359, 1998

6. Park KW, Kim DY, Lee SG, Yoo CJ, Kim WK, Park CW: Longterm results of single level posterior lumbar interbody fusion with stand-alone cage in degenerative. Korean J Spine 4(3):123128, 2007

7. Uzi EA, Dabby D, Tolessa E, Finkelstein JA: Early retropulsion of titanium-threaded cages after posterior lumbar interbody fusion: a report of two cases. Spine 26:1073-1075, 2001

8. Wetzel FT, LaRocca H: The failed posterior lumbar interbody fusion. Spine 16:839-845, 1991 